\title{
Heyde syndrome: gastrointestinal bleeding and aortic stenosis
}

\author{
Bartosz Hudzik MD PhD, Krzysztof Wilczek MD PhD, Mariusz Gasior MD PhD
}

$\mathrm{A}$ n 82-year-old man presented with a twoweek history of increasing shortness of breath and exercise intolerance. His medical history included chronic obstructive pulmonary disease, hypertension and chronic kidney disease. He had undergone left nephrectomy and splenectomy for abdominal trauma and had had three episodes of lower gastrointestinal bleeding for which blood transfusions had been required. Prior workup for gastrointestinal bleeding included colonoscopy and argon plasma coagulation for angiodysplasia.

The patient's vital signs were normal. On chest examination, there were audible crackles at the bases of both lungs and a grade 4/6 systolic murmur at the second right intercostal space radiating toward the right carotid artery. The findings of a rectal examination (including testing for fecal occult blood) were normal. Oxygen saturation was $94 \%$ on room air.

Laboratory tests showed hemoglobin 95 (normal 130-170) g/L, hematocrit 30\% (normal 40\%$52 \%$ ), platelets 201 (normal 150-400) $\times 10^{9} / \mathrm{L}$, creatinine 150 (normal 70-110) $\mu \mathrm{mol} / \mathrm{L}$, estimated glomerular filtration rate 37 (normal > 90) $\mathrm{mL} / \mathrm{min}$ per $1.73 \mathrm{~m}^{2}$ and $N$-terminal pro-B-type natriuretic peptide 5670 (normal < 125) pg/mL. Troponins were within the normal range. Electrocardiography on admission was suggestive of left ventricular hypertrophy with no ischemic changes. Radiographic signs of cardiac enlargement were present. Transthoracic echocardiography showed severe depression of left ventricular systolic function with an ejection fraction of $24 \%$, along with severe aortic stenosis, a heavily calcified aortic valve with aortic valve area of $0.7 \mathrm{~cm}^{2}$ and a mean aortic valve gradient of $37 \mathrm{~mm} \mathrm{Hg}$ (Figure 1).

The history of lower gastrointestinal bleeding (angiodysplasia) and the presence of aortic stenosis were consistent with Heyde syndrome. An enhanced coagulation study was requested, including platelet function and measurement of the function and concentration of von Willebrand complex:factor VIII. The results (prolonged platelet function analyzer [PFA-100] closure time, diminished ristocetin cofactor activity and depletion of high-molecular-weight multimers of von Willebrand factor on gel electrophoresis) confirmed type IIA acquired von Willebrand syndrome (deficit of high-molecular-weight multimers of von Willebrand factor).

Given the patient's comorbidities and an extremely high risk of intraoperative death (logistic EuroSCORE $42.7 \%$ and Society of Thoracic Surgeons adult cardiac surgery risk score 29.3\%; sources available in Appendix 1, at www.cmaj.ca/ lookup/suppl/doi:10.1503/cmaj.150194/-/DC1), the patient was not considered a suitable candidate for surgical aortic valve replacement and was referred for transcatheter aortic valve implantation.

Following implantation of a CoreValve 31 aortic valve, the valve area was $3.7 \mathrm{~cm}^{2}$. The patient did not experience gastrointestinal bleeding in the periprocedural period. However, because of procedural blood loss (bleeding at the access site), two units of packed red blood cells were transfused. The patient was discharged seven days later with a six-month prescription for a single antiplatelet agent (clopidogrel). The 12-month follow-up was unremarkable, with no further episodes of gastrointestinal bleeding reported. Left ventricular ejection fraction had increased to $37 \%$, and the patient's respiratory symptoms had improved (New York Heart Association functional class II). A follow-up coagulation study showed no deficit of high-molecularweight multimers of von Willebrand factor.

\section{KEY POINTS}

- An association exists between severe aortic stenosis and recurrent bleeding from intestinal angiodysplasia.

- For patients with recurrent gastrointestinal bleeding, the differential diagnosis should include Heyde syndrome.

- Management of Heyde syndrome is challenging, with treatment options that include medical therapy, endoscopic interventions, colonic surgery and valve replacement.

- If valve replacement is performed, antiplatelet and anticoagulant agents should be used with caution, to minimize the risk of rebleeding. 


\section{Discussion}

The association between chronic gastrointestinal bleeding due to angiodysplasia and calcific aortic stenosis was first described in 1958 by Edward Heyde and has since been termed Heyde syndrome. Not until 1992 did Warkentin and colleagues elucidate the role of acquired coagulopathy (depletion of high-molecular-weight multimers of von Willebrand factor) in the pathogenesis of Heyde syndrome (historical sources listed in Appendix 1, available at www.cmaj.ca/lookup/ suppl/doi:10.1503/cmaj.150194/-/DC1). Although there is no consensus definition, many authors affirm that Heyde syndrome refers to a triad of aortic stenosis, acquired type IIA von Willebrand syndrome and recurrent bleeding from gastrointestinal angiodysplasia (Figure 2). ${ }^{1-5}$

\section{Epidemiology}

Degenerative aortic stenosis is the most common valvular heart disease in older patients and is one of the main causes of morbidity and mortality in this age group. Its prevalence increases with age, reaching $2 \%-7 \%$ after the age of 65 years. ${ }^{6}$ The presence of symptoms from aortic stenosis heralds poor prognosis and warrants surgery. ${ }^{6}$ However, the role of aortic valve replacement in patients who are asymptomatic remains controversial. ${ }^{6}$

Angiodysplasia is another age-related degenerative process, characterized by dilated, thinwalled tortuous vessels in the mucosa and submucosa of the gastrointestinal tract, including arterioles, capillaries and venules. Angiodysplasia accounts for $1 \%-6 \%$ of hospital admissions for gastrointestinal bleeding. ${ }^{2}$ It may occur throughout the gastrointestinal tract, but most particularly in the right colon and cecum. ${ }^{2,3,7}$

Proving an association between aortic stenosis and angiodysplasia is challenging, because both entities are common in older patients. Some authors have suggested that the rate of aortic stenosis among patients with gastrointestinal bleeding is as high as $30 \%-40 \%{ }^{3}$ Others have reported much lower prevalence (see references available in Appendix 1, www.cmaj.ca/lookup/suppl/ doi:10.1503/cmaj.150194/-/DC1). In patients for whom aortic stenosis is the index diagnosis, there is a 100-fold greater risk of gastrointestinal bleeding, relative to those without aortic stenosis. ${ }^{3}$ Still, angiodysplasia is the most common vascular abnormality of the gastrointestinal tract and the second leading cause (after diverticulosis) of lower gastrointestinal bleeding in patients over the age of $60 .^{7}$

\section{Pathophysiology}

There are several plausible explanations for Heyde syndrome, but the most convincing link between aortic stenosis and gastrointestinal bleeding from angiodysplasia is a deficiency of high-molecularweight multimers of von Willebrand factor. ${ }^{3,4}$ Figure 2 summarizes the pathophysiologic mechanisms underlying Heyde syndrome. Aortic stenosis or sclerosis is associated with high shear stress, which elevates von Willebrand factor-cleaving metalloprotease activity, leading to proteolysis of von Willebrand factor, and increases interactions between von Willebrand factor and platelets, leading to degradation or clearance of von Willebrand factor. ${ }^{4}$

Von Willebrand factor, an adhesive glycoprotein that is synthesized in endothelial cells and megakaryocytes, has two major functions in hemostasis. It helps with platelet-subendothelium adhesion and platelet-platelet interactions (i.e., aggregation), and it carries factor VIII in plasma. Von Willebrand factor also prolongs the half-life of factor VIII by protecting it from proteolytic degradation. It delivers factor VIII to the site of vascular injury, thus enhancing the hemostatic process. ${ }^{8}$ Defects or deficiencies in von Willebrand factor underlie both inherited von Willebrand disease and acquired von Willebrand syndrome. ${ }^{8}$ Changes in the function of von Willebrand factor are not specific to aortic valve disease. Conditions linked to von Willebrand syndrome are listed in Box 1.
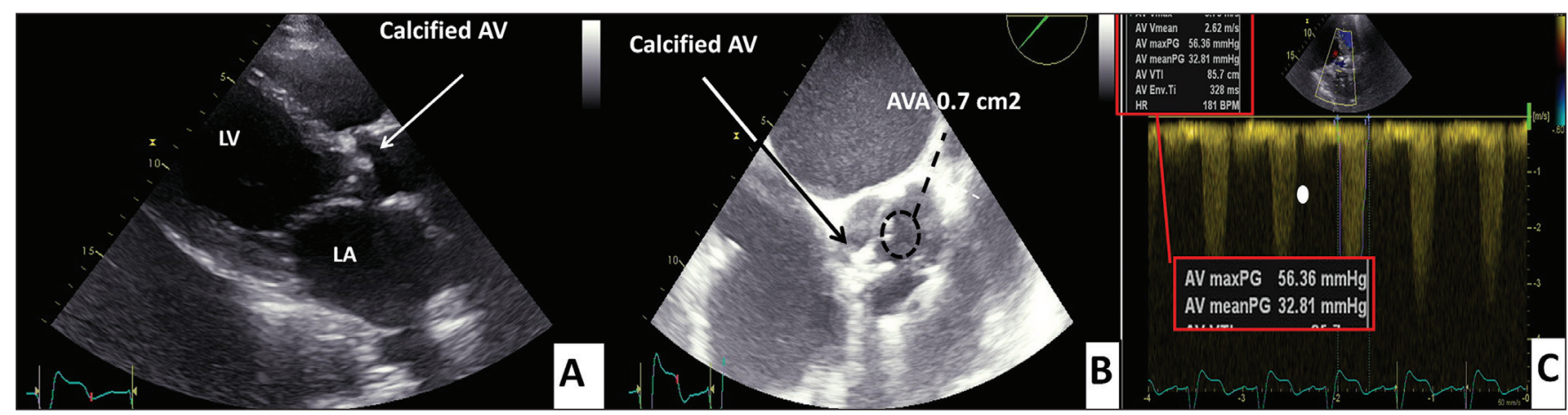

Figure 1: Transthoracic echocardiography shows (A) severe aortic stenosis with heavily calcified aortic valve (AV); (B) aortic valve area (AVA) of $0.7 \mathrm{~cm}^{2}$; and (C) mean aortic valve gradient of $37 \mathrm{~mm} \mathrm{Hg}$. LA = left atrium, LV = left ventricle. 
Other possible factors implicated in the pathogenesis of Heyde syndrome include mucosal ischemia, cholesterol embolization, acquired platelet dysfunction and inflammatory reactions. ${ }^{3}$ Bleeding from angiodysplasia may reflect an interaction between an uncommon coagulopathy (caused by a common valvular disease) and a common vascular disease of the gastrointestinal tract.

\section{Diagnostic workup}

Initial investigations should explore other sources of gastrointestinal bleeding, including gastric or duodenal ulcer, diverticular disease, gastrointestinal malignancy and inflammatory bowel disease. All patients with gastrointestinal bleeding should undergo colonoscopy. Capsule or computed tomography endoscopy may be useful when initial investigations show no abnormality. The presence of angiodysplasia should alert the physician to possible aortic valve disease, and Heyde syndrome should be included in the differential diagnosis for gastrointestinal bleeding in which the cause is unclear. ${ }^{2,3,7}$

Given the complexity of von Willebrand factor pathophysiology, there is no single "robust" assay that can be used in the diagnosis of a bleeding diathesis. Several tests are warranted, which can be categorized as screening tests and discriminating assays (see Appendix 2, www.cmaj.ca/lookup/suppl/ doi:10.1503/cmaj.150194/-/DC1). A complete set of laboratory investigations is necessary to fully diagnose all variants of von Willebrand disease and von Willebrand syndrome. Ranked in descending order, the sensitivity of various tests for type IIA von Willebrand syndrome in patients with bleeding gastrointestinal angiodysplasia is as follows: gel electrophoresis (quantification of high-molecularweight multimers) $>$ PFA-100 closure time $>$ von Willebrand factor ristocetin cofactor $>$ bleeding time $>$ von Willebrand factor antigen. ${ }^{4}$

\section{Treatment}

Management of Heyde syndrome often requires a multidisciplinary approach, and treatment options include medical therapy, endoscopic interventions, colon surgery and aortic valve replacement. ${ }^{3}$ Treatment modalities used in von Willebrand disease (e.g., desmopressin, octreotide or supplementation of factor VIII or von Willebrand factor) are usually insufficient or ineffective for acquired type IIA von Willebrand syndrome. ${ }^{2-4}$ Administration of von Willebrand factor-factor VIII concentrate immediately before surgery should be considered for patients who have transient improvement in von Willebrand factor activity with a test dose (see references listed in Appendix 1). Some experts suggest the use of estrogen-progesterone preparations; however, the mechanism of action of such treatment is still unknown. ${ }^{3,4}$ Thalidomide has been shown to reduce the incidence of severe gastrointestinal bleeding in several studies. ${ }^{3}$ Repeated blood transfusions may offer some, though transient, symptomatic relief.

Local endoscopic interventions for gastrointestinal bleeding include heat probe and bipolarmultipolar coagulation, neodymium-doped yttrium aluminum garnet laser and argon plasma coagulation. ${ }^{3}$ Epinephrine and ethanolamine can be injected independently or concomitantly. Medical or endoscopic interventions for the bowel are often used as bridge therapies to valve

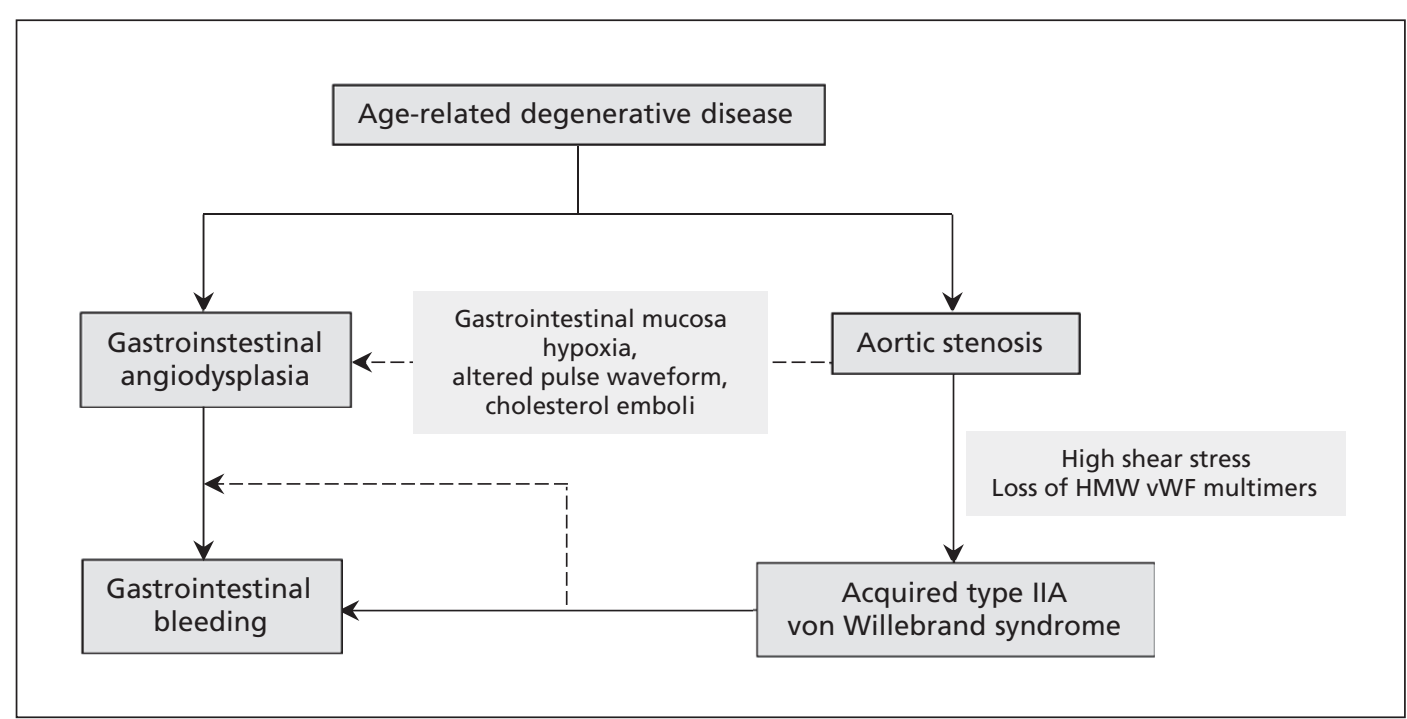

Figure 2: Pathophysiologic mechanisms underlying Heyde syndrome, which link aortic stenosis, gastrointestinal angiodysplasia and acquired type $2 \mathrm{~A}$ von Willebrand syndrome. Solid arrows = recognized mechanisms, dashed arrows = possible mechanisms. Adapted, with permission, from Gola and Lelonek ${ }^{5}(\odot 2010$ Via Medica). HMW = high-molecular-weight, vWF = von Willebrand factor. 
replacement. In cases of severe bleeding, emergent bowel resection may be needed; however, bleeding may continue from other sites. ${ }^{1-3}$ Recurrent severe gastrointestinal bleeding, particularly when medical and endoscopic interventions have failed, often necessitates aortic valve replacement, which is usually employed in the treatment of severe aortic stenosis. ${ }^{1-3}$ Valve replacement has been shown to improve coagulation abnormalities in Heyde syndrome and may offer the possibility of long-term resolution of symptoms. ${ }^{1-3}$

Oral anticoagulation following valve replacement increases the risk of rebleeding. Use of a bioprosthesis precludes long-term anticoagulation. Generally, a bioprosthesis should be considered for patients older than 65 years and is also recommended when anticoagulation at therapeutic levels is contraindicated because of a high bleeding risk

\section{Box 1: Causes of acquired von Willebrand syndrome*}

Cardiovascular disease

- Aortic stenosis

- Hypertrophic cardiomyopathy (with obstruction of left ventricular outflow tract)

- Dysfunctional heart valve prosthesis

- Ventricular septal defect

- Patent ductus arteriosus

- Left ventricular assist device

Autoimmune disorders

- Systemic lupus erythematosus

- Scleroderma

- Mixed connective tissue disease

Lymphoproliferative disorders

- Monoclonal gammopathy of undetermined significance

- Lymphoma

- Multiple myeloma

Myeloproliferative disorders

- Essential thrombocythemia

- Polycythemia vera

- Chronic myeloid leukemia

Nonhematologic neoplasms

- Wilms tumour

- Adenocarcinoma

Drugs

- Ciprofloxacin

- Griseofulvin

- Valproic acid

- Hydroxyethyl starch

- Recombinant factor VIII

Chronic kidney disease (uremia)

Cirrhosis of the liver

*Based on various sources, including Gola and Lelonek ${ }^{5}$ and references listed in Appendix 1 (available at www.cmaj.ca/ lookup/suppl/doi:10.1503/cmaj.150194/-/DC1). (e.g., prior major bleed, comorbidities). ${ }^{6}$ Experts recommend lifelong oral anticoagulation for patients with a mechanical prosthesis. ${ }^{6}$ Low-dose acetylsalicylic acid (ASA) should be considered for the first three months after implantation of an aortic bioprosthesis. ${ }^{6}$ Alternatively, oral anticoagulation may be considered for the first three months after implantation of an aortic bioprosthesis. ${ }^{6}$

More recently, transcatheter aortic valve implantation has emerged as a feasible option for patients with severe aortic stenosis whose risk is deemed too high for surgical aortic valve replacement. ${ }^{9}$ Experts recommend dual-antiplatelet therapy (low-dose ASA + clopidogrel) for one to three months following the procedure. ${ }^{10}$ However, the use of ASA could increase the risk of recurrent gastrointestinal bleeding. A recent systematic review and meta-analysis ${ }^{11}$ showed that dual-antiplatelet therapy following transcatheter aortic valve implantation had no benefit over single-antiplatelet therapy in terms of reduction of ischemic events, with a trend toward increased harm due to bleeding. The authors concluded that in future, consideration should be given to clopidogrel monotherapy.

\section{References}

1. Massyn MW, Khan SA. Heyde syndrome: a common diagnosis in older patients with severe aortic stenosis. Age Ageing 2009; 38:267-70, discussion 251.

2. Pate GE, Chandavimol M, Naiman SC, et al. Heyde's syndrome: a review. J Heart Valve Dis 2004;13:701-12.

3. Islam S, Cevik C, Islam E, et al. Heyde's syndrome: a critical review of the literature. J Heart Valve Dis 2011;20:366-75.

4. Warkentin TE, Moore JC, Anand SS, et al. Gastrointestinal bleeding, angiodysplasia, cardiovascular disease, and acquired von Willebrand syndrome. Transfus Med Rev 2003;17:272-86.

5. Gola W, Lelonek M. Clinical implication of gastrointestinal bleeding in degenerative aortic stenosis: an update. Cardiol $J$ 2010;17:330-4.

6. Vahanian A, Alfieri O, Andreotti F, et al. Guidelines on the management of valvular heart disease (version 2012). Eur Heart J 2012;33:2451-96.

7. Regula J, Wronska E, Pachlewski J. Vascular lesions of the gastrointestinal tract. Best Pract Res Clin Gastroenterol 2008; 22:313-28.

8. Flood VH. Perils, problems, and progress in laboratory diagnosis of von Willebrand disease. Semin Thromb Hemost 2014;40:41-8.

9. Godino C, Lauretta L, Pavon AG, et al. Heyde's syndrome incidence and outcome in patients undergoing transcatheter aortic valve implantation. J Am Coll Cardiol 2013;61:687-9.

10. Webb J, Rodes-Cabau J, Fremes S, et al. Transcatheter aortic valve implantation: a Canadian Cardiovascular Society position statement. Can J Cardiol 2012;28:520-8.

11. Gandhi S, Schwalm JDR, Velianou JL, et al. Comparison of dual-antiplatelet therapy to mono-antiplatelet therapy after transcatheter aortic valve implantation: systematic review and metaanalysis. Can J Cardiol 2015;31:775-84.

Affiliation: Third Department of Cardiology, Silesian Centre for Heart Disease, Medical University of Silesia, Zabrze, Poland

Contributors: Both authors made substantial contributions to the conception of the manuscript and to drafting and revising the manuscript, gave final approval of the revised version to be published and agree to act as guarantors for the work.

Acknowledgement: The authors acknowledge Dr. Lukasz Siedlecki for his contribution in the preparation of the manuscript. 